\title{
Tratamiento de la artrosis postraumática de la interfalángica proximal mediante artroplastia de carbono pirolítico
}

\author{
J. Mota da Costa ${ }^{(1)}$, A. Pinto ${ }^{(2)}$ \\ HOSPITAL CUF DESCOBERTAS \\ (1)Asistente Hospitalario Graduado de Cirugía Plástica y Reconstructiva \\ ${ }^{(2)}$ Asistente Hospitalario de Cirugía Plástica Y ReConstructiva
}

Correspondencia:

Dr. João Mota da Costa

Hospital Cuf Descobertas

Rua Mário Botas,1998-018

Lisboa - Portugal

e-mail: joao.costa@hospitalcufdescobertas.pt

Objetivos: Valorar los resultados obtenidos mediante la artroplastia de carbono pirolítico en pacientes con artrosis postraumática de las interfalángicas proximales.

Material y Método: Se analizan un total de 25 pacientes (24 hombres, 1 mujer) con artrosis postraumática de la articulación interfalángica proximal (IFP), que fueron valorados clínica y radiográficamente, después de haber sido intervenidos mediante una artroplastia de carbono pirolítico. Todos los casos fueron operados por vía dorsal, siguiendo siempre el mismo protocolo rehabilitador postquirúrgico. El seguimiento medio de estos pacientes fue de 42 meses, con un mínimo de 12 meses y un máximo de 72 meses.

Resultados: Comparando la escala analógica de dolor y el rango de movilidad en el pre y postoperatorio, se obtuvieron en 24 casos, unos buenos resultados. El único caso que fue considerado como malo, presentó una subluxación dorsal del componente distal del implante, que requirió de una reducción quirúrgica. En cuanto a los hallazgos radiográficos, en el $10 \%$ de los casos se objetivaron finas bandas radiolúcidas peri-implante, si bien no se correlacionó este hallazgo con un deterioro clínico.
Objectives: To measure the results obtained using a pyrocarbon arthroplasty in patients with post-traumatic arthrosis of the proximal interphalangeal joints.

Materials and Methods: Twenty five patients (24 male, 1 female) with post-traumatic arthrosis of the proximal interphalangeal joints were studied, with a clinical and radiological assessment after undergoing a pyrocarbon arthroplasty. All cases were operated through a dorsal approach and underwent the same post-operative regime. Average follow-up was 42 months, range 12 months to 72 months.

Results: The change in the analog pain scale and range of movement pre and pot-operative indicates that the results were good in 24 cases. The only result considered poor presented a dorsal subluxation of the implant, requiring surgical reduction. Radiologically, $10 \%$ of cases show fine radiolucent lines around the implant, but this finding did not correlate with a clinical deterioration.

Conclusions: Replacement arthroplasty of the PIPJ using a pyrocarbon implant, presents a valid alternative in cases with post-traumatic arthrosis. However, larger series with longer follow up are required to validate this treatment. 
Conclusiones: La artroplastia de sustitución de la articulación IFP mediante un implante de carbono pirolítico, constituye una alternativa muy válida en los casos de artrosis postraumática. Sin embargo, es preciso contar son series más largas y con más tiempo de evolución para validar este tipo de tratamiento.

Palabras clave: IFP, artroplastia, artrosis, carbono pirolítico.

Key Words: PIPJ, arthroplasty, arthrosis, pyrocarbon

Rev. Iberam. Cir. Mano - Vol. 35 - Núm. 1 Mayo 2007 (38-42)

\section{INTRODUCCIÓN}

La artrosis postraumática de la articulación interfalángica proximal (IFP) de los dedos de la mano, constituye un verdadero reto terapéutico, pues no existe, de momento, una solución definitiva. En este sentido, el tratamiento tradicional de esta particular patología, ha consistido en la artrodesis y/o la artroplastia de sustitución mediante implantes de silicona flexible ${ }^{1-2}$.

Por lo que respecta a la artrodesis, ha sido y es, el tratamiento de elección de la artrosis postraumática de la IFP, especialmente en pacientes con trabajos pesados, pues proporciona una gran estabilidad, corrige la deformidad y elimina el dolor. Sin embargo, presenta el gran inconveniente de eliminar el movimiento y afectar considerablemente la prensión de la mano, fundamentalmente cuando se realiza en los dedos cubitales ${ }^{1}$. En cuanto al implante de silicona flexible, si bien ofrece la ventaja de no sacrificar el movimiento, presenta varios problemas como son la deformidad angular en el caso de una mala resección ósea, la rotura del implante, la erosión cortical y la sinovitis por partículas de silicona, entre otros ${ }^{2-3}$.

Vistos los inconvenientes planteados con ambas intervenciones, se empezaron a desarrollar



Figura 1. Artroplastia de la articulación IFP con material de carbono pirolítico. otro tipo de técnicas, entre las que cabe destacar las artroplastias, ya sean con interfase me$\mathrm{tal} / \mathrm{metal}$, metal/polietileno o mediante materiales viscoelásticos, con o sin la utilización de cemento acrílico. Aunque los primeros resultados con estos nuevos tipos de artroplastia eran muy esperanzadores, pronto se observó también la aparición de complicaciones, como son los aflojamientos y las erosiones corticales alrededor de los implantes ${ }^{4-5}$.

La última generación de artroplastias de la IFP, se construyen con biomateriales que resisten adecuadamente las fuerzas de cizallamiento $y$, en consecuencia, minimizan su desgaste. Entre ellas, cabe citar las de carbono pirolítico (Ascensión Orthopaedics, Austin TX, diseñadas por John Stanley y Robert Beckenbaugh), que son semiconstreñidas, bicondíleas y con retención de los ligamentos colaterales (Figura 1). Si bien se empezaron a utilizar en Europa en el año 2000. Aún no existen series a largo plazo con este tipo de implante que indiquen claramente que su utilización aporten más ventajas que sus predecesoras y/o que superen los beneficios de la artrodesis. Es por ello, que nos pareció interesante, en este trabajo, valorar los resultados obtenidos en una serie de pacientes con artrosis postraumática de la IFP, a los que se les colocó una artroplastia de carbono pirolítico.

\section{MATERIAL Y MÉTODO}

Se han analizado 25 pacientes con 26 artroplastias de carbono pirolítico de las IFP, de los que 24 eran varones y 1 mujer, con edades comprendidas entre 32 y 58 años (media: 41 años). 




Figura 2. Estudio radiográfico de las secuelas de la articulación IFP después de una fractura intracondílea de la falange proximal. A) proyección lateral. B) proyección anteroposterior.

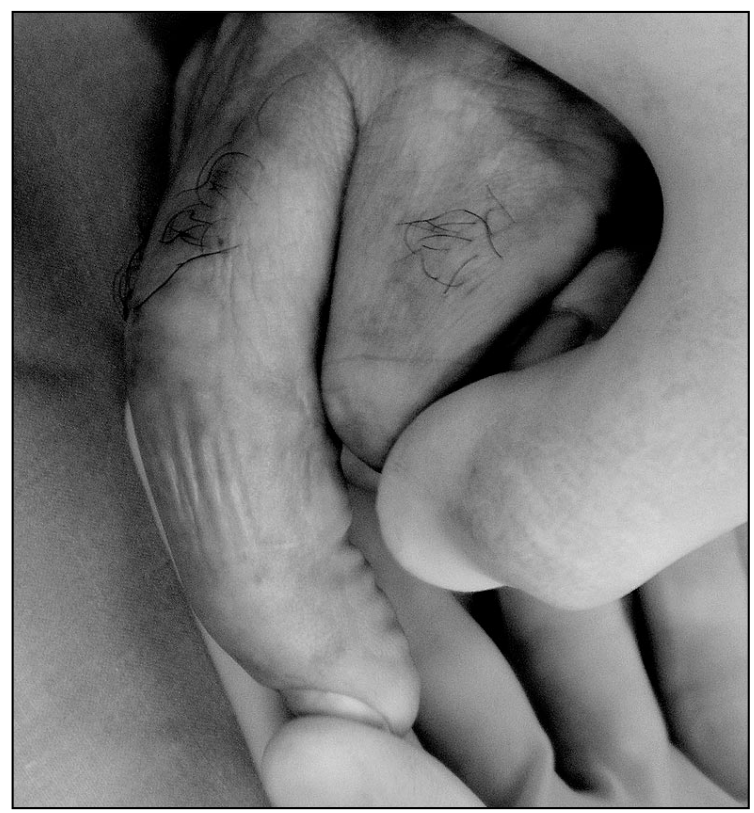

Figura 3. Rigidez postraumática, sin fractura, de la IFP. Obsérvese el mínimo grado de flexión pasiva que conseguía el paciente.

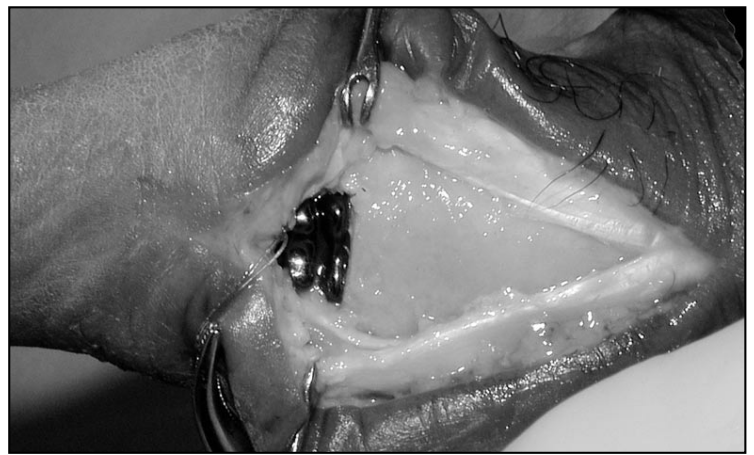

Figura 4. Abordaje de la articulación IFP mediante incisión longitudinal de la bandeleta central del tendón extensor.

El seguimiento medio de estos pacientes fue de 42 meses (mínimo: 12 meses; máximo: 72 meses), siendo los dedos afectados: Indice (5), Medio (7), Anular (8) y meñique (6). La patogenia de la artrosis fue traumática (fracturas articulares no tratadas o con tratamiento insuficiente) en 17 casos (Figura 2) y en $\operatorname{los} 8$ casos restantes como consecuencia de una rigidez dolorosa (Figura 3), de origen también traumático, pero sin lesiones osteoarticulares.

En cuanto a la técnica quirúrgica, en todos los casos se utilizó la anestesia troncular, fundamentalmente axilar. La incisión fue siempre dorsal, con división longitudinal del tendón extensor en 19 casos (Figura 4) o con colgajo de base distal del mismo tipo Chamay ${ }^{6}$ en 7 (Figura 5). El tipo de inmovilización postoperatoria consistió en un vendaje enyesado de muñeca y mano, manteniendo las interfalángicas (IFs) en extensión y las metacarpofalángicas (MCFs) en flexión de 20․ El protocolo seguido para la rehabilitación, en los casos en los que se abordó la articulación con incisión longitudinal del extensor, se inició al 4º día postoperatorio, con el uso de una férula estática



Figura 5. Abordaje de la articulación IFP mediante la creación de un colgajo de base distal del tendón extensor. 


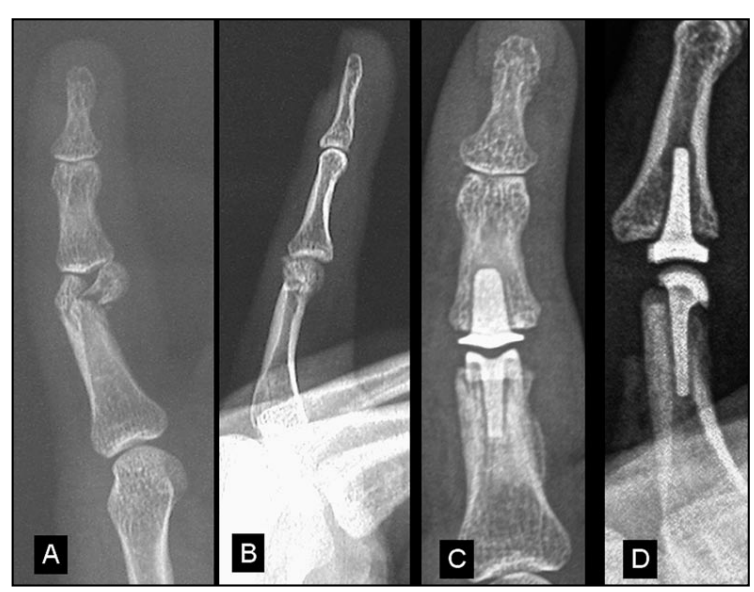

Figura 6. Estudio radiográfico de una artroplastia de carbón pirolítico en un paciente con artrosis postraumática de la IFP del dedo meñique. A) proyección anteroposterior preoperatoria. B) proyección lateral preoperatoria. C) proyección anteroposterior postoperatoria. D) proyección lateral postoperatoria.

posicional nocturna y una férula dinámica durante el resto del día, con lo que se permitía la flexión activa y libre de las articulaciones MCFs e IFs, y la extensión pasiva asistida por la ortesis. Se instruía al paciente para que no sobrepasase la flexión de las IF en más de $30^{\circ}$ durante la primera semana, hasta llegar, progresivamente, a los $75^{\circ}$ o más, durante las 4 semanas siguientes. En los casos en los que se abordó la articulación mediante colgajo del extensor de base distal, la inmovilización postoperatoria se prolongó durante 2 semanas, siguiendo después con el mismo protocolo ya comentado.

En el seguimiento clínico de estos pacientes, se valoró el grado de dolor según la esca- la analógica visual y el rango de movimiento, mientras que en el seguimiento radiográfico se prestó una especial atención sobre la existencia o no de subluxación de los componentes de la artroplastia y la presencia de imágenes indicativas de aflojamiento. Los valores de los dos primeros parámetros (dolor y movilidad) se compararon con los obtenidos al final del estudio.

\section{RESULTADOS}

Valorando únicamente el dato subjetivo del dolor, 24 de los 25 pacientes se mostraron satisfechos con el resultado y solamente en un caso, el paciente no notó ninguna mejoría. Dicho caso, requirió de una segunda intervención por la subluxación del implante. En el resto de las artroplastias, no se objetivaron desaxaciones entre sus componentes ni inestabilidades angulares (Figura 6), aunque sí se pudo observar en el $10 \%$ de ellos, una fina banda radiolúcida peri-implante, indicativo de aflojamiento, si bien no se correlacionó dicho hallazgo con un deterioro clínico, al menos durante el tiempo que duró este trabajo.

En cuanto al movimiento, se pasó de un rango medio de $14^{\circ}\left(0^{\circ}-38^{\circ}\right)$ en el preoperatorio, a un rango medio de $70^{\circ}\left(-5^{\circ}-84^{\circ}\right)$ al final del estudio (Figura 7).

No hubo que lamentar complicaciones de tipo infeccioso, nervioso y/o de síndrome doloroso regional complejo.

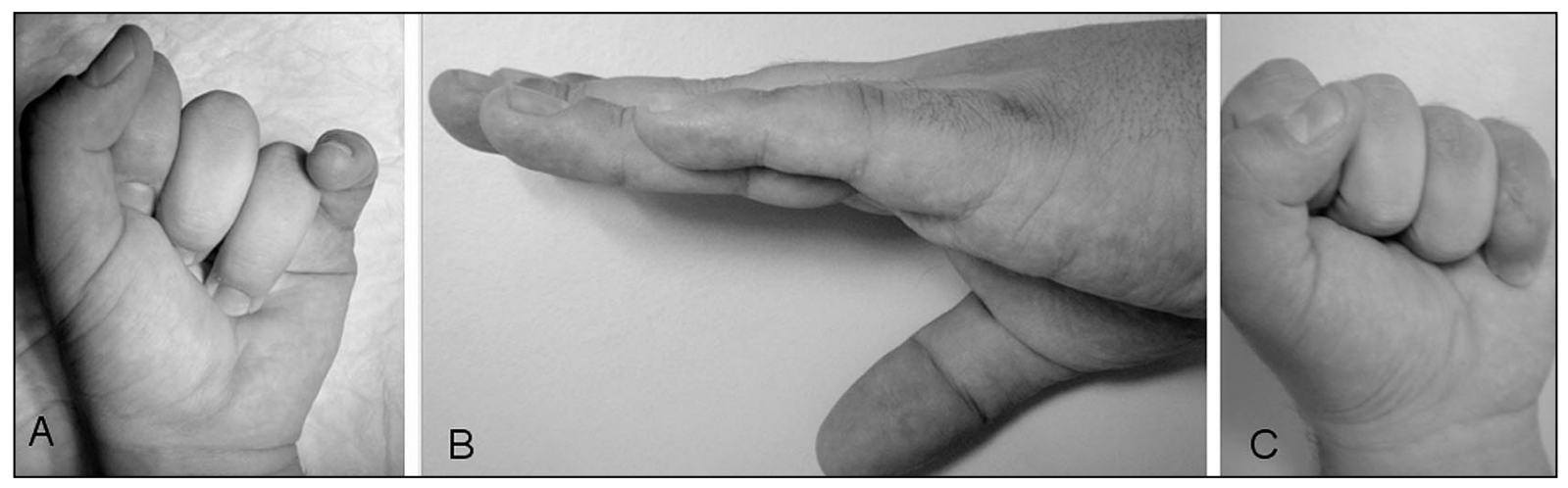

Figura 7. Resultado clínico del mismo paciente de la figura 6. A) movilidad preoperatoria. B) extensión. C) flexión. Obsérvese que en este caso se ha conseguido un rango de movilidad total. 


\section{DISCUSIÓN}

Tradicionalmente, aún se considera la artrodesis como el tratamiento de elección en la artrosis postraumática de la IFP. En este sentido, existen varias publicaciones que avalan dicho proceder en esta patología al lograr un gran porcentaje de casos con buenos resultados ${ }^{7}$. En cuanto a las artroplastias de sustitución con implantes de silicona flexible, y también aplicadas a la artrosis postraumática, los resultados son controvertidos. Así, mientras que Takigawa et al. ${ }^{8}$, obtienen malos resultados en más de la mitad de sus pacientes, Mathoulin y Gilbert ${ }^{9}$ muestran un $60 \%$ de buenos resultados, recomendando formalmente su utilización para este tipo de patología, pues no encuentran que las demás técnicas existentes para tratar la misma, superen sus resultados. Y con respecto a las artroplas- tias de carbono pirolítico, también hay diferencias considerables según el autor consultado. Así, mientras que Nunley et al. ${ }^{10}$, abandonaron prematuramente un trabajo clínico prospectivo con este tipo de artroplastias al obtener en sus primeros 5 casos unos muy malos resultados y con complicaciones añadidas, y que Tuttle y Stern ${ }^{11}$ consideraron dicho procedimiento como impredecible, en cuanto a resultados se refiere, tenemos a Schulz et al. ${ }^{12}$, quienes en un estudio retrospectivo sobre 20 pacientes con artrosis postraumática y con un seguimiento medio entre 6 meses y 3 años, consideran que la artroplastia de carbono pirolítico es una buena opción de tratamiento para este tipo de casos. Nuestro trabajo puede equipararse a este último, si bien apuntamos también, la necesidad de un estudio con más número de casos y a más largo plazo para sacar conclusiones válidas al respecto.

\section{BIBLIOGRAFÍA}

1. Jones BF, Stern PJ. Interphalangeal joint arthrodesis. Hand Clin, 1994; 10: 267-75.

2. Swanson AB, Maupin BK, Gajjar NV, Swanson GD. Flexible implant arthroplasty in the proximal interphalangeal joint of the hand. J Hand Surg Am, 1985; 10: 796-805.

3. Reis ND, Calnan JS. Integral hinge joint. Ann Rheum Dis, 1969; 28 (suppl): 59-62.

4. Dryer RF, Blair WF, Shurr DG, Buckwalter JA. Proximal interphalangeal joint arthroplasty. Clin Orthop, 1984; 185: 18794.

5. Linscheid RL, Dobyns JH. Total joint arthroplasty. The Hand. Mayo Clin Proc, 1979; 54: 51626.
6. Chamay A. A distally based dorsal and triangular tendinous flap for direct access to the proximal interphalangeal joint. Ann Chir Main, 1988; 7: 17983.

7. Stern PJ, Gates NT, Jones TB. Tension band arthrodesis of small joints in the hand. J Hand Surg Am, 1993; 18: 194-7.

8. Takigawa S, Meletiou S, Sauerbier M, Cooney WP. Long-term assessment of Swanson implant arthroplasty in the proximal interphalangeal joint of the hand. J Hand Surg Am, 2004; 29: 785-95.

9. Mathoulin C, Gilbert A. Arthroplasty of the proximal interphalangeal joint using the Sutter implant for traumatic joint des- truction. J Hand Surg Br, 1999; 24: 565-9.

10. Nunley RM, Boyer MI, Goldfarb CA. Pyrolitic carbon arthroplasty for posttraumatic arthritis of the proximal interphalangeal joint. J Hand Surg Am, 2006; 31: 1468-74.

11. Tuttle HG, Stern PJ. Pyrolitic carbon proximal interphalangeal joint resurfacing arthroplasty. J Hand Surg Am, 2006; 31: 930-9.

12. Schulz M, Muller-Zimmermann $A$, Behrend $M$, Krimmer $\mathrm{H}$. Early results of proximal interphalangeal joint replacement with pyrolitic carbon prosthesis (Ascension) in idiopathic and post-traumatic arthritis. Handchir Mikrochir Plast Chir, 2005; 37: 26-34. 